\title{
A Low-Frequency Annular-Slot Antenna
}

\author{
James R. Wait
}

The radiation characteristics of an annular slot cut in an ideally conducting ground plane are discussed. The voltage impressed between the concentric edges is assumed to be constant around the slot. The annular slot is backed by a hemispherical cavity which has imperfectly conducting walls. For a specified voltage, the power radiated in the upper half-space and the power absorbed by the hemispherical cavity are calculated. It is indicated that the power absorbed can be reduced greatly by lining the walls of the cavity with a wire mesh. A flush-mounted antenna of this type at low frequencies may have certain practical advantages over the more conventional monopole.

It is the purpose of this paper to examine the radiation characteristics of an annular slot cut in a thin perfectly conducting ground plane. The medium below the ground plane is taken to be a dissipative medium such as soil. A hemispherical bowl is hollowed out just beneath the annular slot. The problem is to calculate the power radiated into the upper half-space and the power absorbed by the walls of the hemispherical cavity.

The radiation into a half-space from an aperture or a slot cut in a perfectly conducting plane of infinite extent is derivable from a knowledge of the tangential electric field distribution $\vec{E}^{\prime}$ over the aperture or slot. A general expression for the magnetic field $\vec{H}$ in the exterior region $[1]^{1}$ is (for a time factor $\exp (i \omega t)$ )

$$
\vec{H}(\vec{r})=\frac{i k}{\eta_{0}} \iint_{\text {slot }} \vec{n} \times \vec{E}^{\prime} \cdot \Gamma\left(\vec{r}, \overrightarrow{r^{\prime}}\right) d s,
$$

where $k=2 \pi /$ wavelength, $\eta_{0}=120 \pi, \vec{n}$ is a unit vector which is pointing out and is normal to the slot aperture, $\vec{r}$ and $\overrightarrow{r^{\prime}}$ are position vectors directed to a point in the aperture and to the field point respectively. $\quad \Gamma\left(\vec{r}, \overrightarrow{r^{\prime}}\right)$ is the dyadic Green's function and is given by the operator

$$
\Gamma\left(\vec{r}, \overrightarrow{r^{\prime}}\right) \cdot=\left(I+\frac{1}{k^{2}} \nabla \nabla\right) G\left(\overrightarrow{r,} \vec{r}^{\prime}\right)
$$

in terms of the scalar Green's function $G\left(\vec{r}, \overrightarrow{r^{\prime}}\right)$ which, for a halfspace, is

$$
G\left(\overrightarrow{r, r^{\prime}}\right)=\frac{1}{2 \pi} \frac{e^{-i k\left|\vec{r}-\vec{r}^{\prime}\right|}}{\left|\vec{r}-\overrightarrow{r^{\prime}}\right|}
$$

Equation (1) can readily be applied to the case of an annular slot cut in a flat ground plane (see fig. 1). A spherical coordinate $\operatorname{system}(r, \theta, \phi)$ is chosen with the ground plane defined by $\theta=\pi / 2$ and the inner and outer rim of the annular slot are $r=b-\Delta$ and $r=b+\Delta$, respectively. It is now assumed that the field in the slot aperture has only a radial component $E^{\prime}\left(\rho^{\prime}\right)$ which does not vary in the $\phi$ direction. The resultant magnetic field in the exterior half-space has only a $\phi$ component and is given by

$$
H_{\phi}=\frac{i k}{2 \pi \eta_{0}} \int_{\phi=0}^{2 \pi} \int_{\rho=b-\Delta}^{b+\Delta} \rho^{\prime} \cos \phi^{\prime} \frac{e^{-i k \mid \vec{r}-\overrightarrow{\rho^{\prime} \mid}}}{\vec{r}-\overrightarrow{\rho^{\prime}} \mid} E\left(\rho^{\prime}\right) d \rho^{\prime} d \phi^{\prime}
$$

${ }_{1}^{1}$ Figures in brackets indicate the literature references at the end of this paper. 


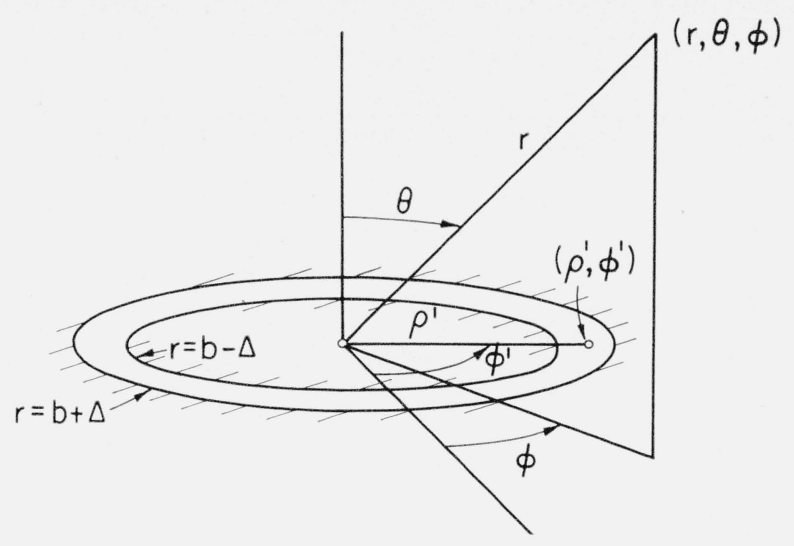

Figure 1. Annular slot and upper half-space.

where $\vec{\rho}$ is a position vector to a point in the slot. For a narrow slot such that $\Delta$ is small compared to $b$ and to the wavelength, the above simplifies to

$$
H_{\phi} \simeq \frac{i k b}{2 \pi \eta_{0}} V \int_{0}^{2 \pi} \cos \phi^{\prime} \frac{e^{-i k R}}{R} d \phi^{\prime},
$$

where $R=\left[r^{2}+b^{2}-2 r b \sin \theta \cos \phi^{\prime}\right]^{1 / 2}$ and

is the voltage across the slot.

$$
V=\frac{1}{b} \int_{b-\Delta}^{b+\Delta} E\left(\rho^{\prime}\right) \rho^{\prime} d \rho^{\prime}
$$

In that far field, $k r>>1$ and therefore $k R \cong k\left[r-b \sin \theta \cos \phi^{\prime}\right]$ so that

$$
\begin{aligned}
H_{\phi} & =\frac{i k b V}{2 \pi \eta_{0}} \frac{e^{-i k r}}{r} \int_{0}^{2 \pi} \cos \phi^{\prime} e^{i k b \sin \theta \cos \phi^{\prime}} d \phi^{\prime} \\
& =-\frac{k b V}{\eta_{0}} \frac{e^{-i k r}}{r} J_{1}(k b \sin \theta)
\end{aligned}
$$

where $J_{1}(x)$ is the Bessel function of order one. For $k b<<1$,

$$
H_{\phi} \simeq-\frac{(k b)^{2} V}{2 \eta_{0}} \frac{e^{-i k r}}{r} \sin \theta
$$

which has the same radiation pattern as a vertical electric dipole.

The power, $P_{r}$, radiated into the upper half-space is obtained by integrating the Poynting vector over an infinite hemisphere, therefore

$$
P_{r}=\lim _{r \rightarrow \infty} \frac{\eta_{0}}{2} \int_{0}^{\pi / 2}\left|H_{\phi}\right|^{2}(2 \pi r \sin \theta) d \theta
$$

and consequently the radiation conductance, $g_{\tau}$, for the upper half-space is ${ }^{2}$

${ }^{2}$ It may be of interest to note that $y_{\nabla}$ can be expressed in the alternate form

$$
g_{r}=\frac{\pi}{4 k b} \int_{0}^{2 k b} J_{2}(2 x) d x .
$$

This integral has been tabulated by H. L. Knudsen in Antenna "systems with rotational symmetry, D. Sc. thesis (Tech. Univ. of Denmark, Copenhagen, 1953). 


$$
g_{r}=\frac{2 P_{r}}{V^{2}}=\frac{2 \pi(k b)^{2}}{\eta_{0}} \int_{0}^{\pi / 2} J_{1}^{2}(k b \sin \theta) \sin \theta d \theta .
$$

'This integral can be written in terms of a highly convergent series as was shown by Bailey [2]; consequently

For $k b<<1$,

$$
g_{r}=\frac{2 \pi k b}{\eta_{0}} \sum_{m=0,1,2 \ldots}^{\infty} J_{3+2 m}(2 k b) .
$$

$$
g_{r} \cong \frac{\pi}{\eta_{0}} \frac{(k b)^{4}}{6}
$$

In the preceding discussion, only the upper half-space has been considered. If the annular slot was cut in a metal plate located in free space, the total radiation conductance would be simply $2 g_{r}$. In the present case, however, the annular slot is to be backed by a hemispherical cavity of radius $a$ as indicated in figure 2. The magnetic field $H_{\phi}$ in the cavity consists of two parts, the primary field $H_{\phi}^{p}$ and the secondary field $H_{\phi}^{s}$. The tangential fields at the walls of the cavity, which consist of "lossy" material, are assumed to satisfy the approximate boundary condition $E_{\theta}=\left.Z H_{\phi}\right|_{r=a}$, where $Z$ is the surface impedance. ${ }^{3}$ The solution for the fields in the cavity can be obtained quite readily by using spherical wave functions.

The primary field is, of course,

$$
H_{\phi}^{p}=\frac{i k b V}{2 \pi \eta_{0}} \int_{0}^{2 \pi} \cos \theta^{\prime} \frac{e^{-i k R}}{R} d \phi^{\prime}
$$

It is now noted that [3,p.1466]

$$
\begin{gathered}
\frac{e^{-i k R}}{R}=-i k \sum_{n=0}^{\infty} \sum_{m=0}^{n}(2 n+1) \epsilon_{m} \frac{(n-m) !}{(n+m) !} \cos m\left(\phi-\phi^{\prime}\right) . \\
P_{n}^{m}(0) P_{n}^{m}(\cos \theta) \begin{cases}j_{n}(k b) h_{n}(k r) ; & r>b \\
j_{n}(k r) h_{n}(k b) ; & r<b\end{cases}
\end{gathered}
$$

where $P_{n}^{m}(\cos \theta)$ is the associated Legendre polynomial and $j_{n}(x)$ and $h_{n}(x)$ are spherical Bessel and Hankel functions, respectively. ${ }^{4}$ As a consequence of the orthogonality, the terms for

3 The use of surface impedance is valid when tho fields in the air-filled cavity vary slowly in a distance equal to the "skin depth" of the lossy material beyond the cavity walls. This is satisfied if $Z / \eta_{0}<<1$.

1 The spherical Bessel and Hankel functions are defined by

$$
\begin{aligned}
& j_{n}(x)=(-1)^{n} x^{n}\left(\frac{d}{x d x}\right)^{n}\left(\frac{\sin x}{x}\right) \\
& h_{n}(x)=i(-1)^{n} x^{n}\left(\frac{d}{x d x}\right)^{n}\left(\frac{e^{-i x}}{x}\right) .
\end{aligned}
$$

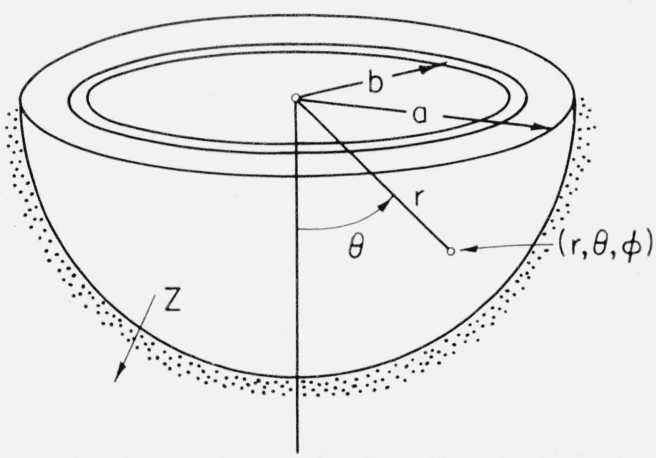

Figure 2. Annular slot and lower hemisphere. 
$m \neq 1$ vanish in the integration with respect to $\phi^{\prime}$, therefore

$$
H_{\phi}^{p}=\sum_{n=1}^{\infty} A_{n} h_{n}(k r) P_{n}^{1}(\cos \theta)
$$

for $r>b$, where

$$
A_{n}=\frac{k^{2} b V}{\eta_{0}} \frac{2 n+1}{n(n+1)} P_{n}^{1}(0) j_{n}(k b) .
$$

The secondary field within the cavity is finite at $r=0$, is a solution of the wave equation, has azimuthal symmetry, and is therefore of the form

$$
H_{\phi}^{s}=\sum_{n=1}^{\infty} B_{n} j_{n}(k r) P_{n}^{1}(\cos \theta)
$$

The tangential electric field is obtained from

$$
E_{\theta}=-\frac{1}{i \epsilon \omega r} \frac{\partial}{\partial r}\left(r H_{\phi}\right)
$$

so that

$$
E_{\theta}=\frac{i \eta_{0}}{k r} \sum_{n=1}^{\infty}\left\{A_{n} \frac{\partial}{\partial r}\left[r h_{n}(k r)\right]+B_{n} \frac{\partial}{\partial r}\left[r j_{n}(k r)\right]\right\} P_{n}^{1}(\cos \theta) .
$$

Application of the boundary condition at $r=a$, leads to

where

$$
\frac{B_{n}}{A_{n}}=-\frac{h_{n}^{\prime}+\left(i Z / \eta_{0}\right) h_{n}}{j_{n}^{\prime}+\left(i Z / \eta_{0}\right) \dot{j}_{n}}
$$

$$
\begin{aligned}
& h_{n}=h_{n}(k a), j_{n}=j_{n}(k a), \\
& h_{n}^{\prime}=\left\{\frac{1}{x} \frac{\partial}{\partial x}\left[x h_{n}(x)\right]\right\}_{x=k a}, \\
& j_{n}^{\prime}=\left\{\frac{1}{x} \frac{\partial}{\partial x}\left[x j_{n}(x)\right]\right\}_{x=k a} .
\end{aligned}
$$

Explicit expressions for the fields inside the cavity are thus available. The power absorbed, $P_{a}$, by the lossy walls can be obtained by integrating the Poynting flux crossing the hemisphere at $r=a$. Making use of the Wronskian relation [3, p. 1573],

it readily follows that

$$
h_{n}(x) \frac{d j_{n}(x)}{d x}-j_{n}(x) \frac{d h_{n}(x)}{d x}=\frac{i}{x^{2}}
$$

$$
\left.H_{\phi}\right]_{r=a}=\sum_{n=1}^{\infty} S_{n} P_{n}^{1}(\cos \theta)
$$

where

$$
S_{n}=\frac{i A_{n}}{(k a)^{2}} \frac{1}{j_{n}^{\prime}+(i Z / \eta) j_{n}} .
$$

Therefore $^{5}$

$$
2 P_{a}=\left[2 \pi \operatorname{Re} Z \int_{0}^{\pi / 2}\left|H_{\phi}\right|^{2} \sin \theta r^{2} d \theta\right]_{r=a}
$$

and

$$
\frac{2 P_{a}}{\operatorname{Re} Z}=2 \pi a^{2} \sum_{n=1}^{\infty} \sum_{n^{\prime}=1}^{\infty} S_{n} S_{n^{\prime}}^{*} \int_{0}^{\pi / 2} P_{n}^{1}(\cos \theta) P_{n^{\prime}}^{1}(\cos \theta) \sin \theta d \theta .
$$


Noting that $[3$, p. 783 , part 1$]$

it follows that

$$
\begin{aligned}
\int_{-1}^{+1} P_{n}^{m}(x) P_{n^{\prime}}^{m}(x) d x & =\frac{2}{2 n+1} \frac{(n+m) !}{(n-m) !} \text { for } n=n^{\prime} \\
& =0 \text { for } n \neq n^{\prime}
\end{aligned}
$$

$$
\frac{2 P_{a}}{\operatorname{Re} Z}=2 \pi a^{2} \sum_{n=1}^{\infty} S_{n} S_{n}^{*} \cdot \frac{n(n+1)}{2 n+1}
$$

where the asterisk denotes a complex conjugate. The conductance as seen by the annular slot, for the lower hemispherical bowl, is then given by ${ }^{6}$

$$
\begin{aligned}
g_{a} & =\frac{2 P_{a}}{V^{2}} \\
& =\frac{2 \pi \operatorname{Re} Z}{\eta_{0}^{2}}\left(\frac{b}{a}\right)^{2} \sum_{n=1}^{\infty} \frac{2 n+1}{n(n+1)} \frac{\left[P_{n}^{1}(0)\right]^{2}\left[j_{n}(k b)\right]^{2}}{\left|j_{n}^{\prime}+\left(i Z / \eta_{0}\right) j_{n}\right|^{2}} .
\end{aligned}
$$

The total conductance at the annular slot is then $g_{a}+g_{r}$. The efficiency, $\xi$, of the annular slot can be defined as the ratio of the power radiated into the upper half-space to the total power, therefore,

$$
\xi=\frac{P_{r}}{P_{a}+P_{r}}=\frac{1}{\left(g_{a} / g_{r}\right)+1} .
$$

The preceding formulas could be employed to calculate the efficiency of the antenna for given values of the surface impedance $Z$, the radius of the annular ring, and the radius of the hemispherical cavity. Such an antenna might be feasible even at low radiofrequencies. In this case, the cavity could be a canyon, gorge, or ditch. Using a system of wires stretched between the canyon rims, a simulated annular aperture could be fabricated. In such an application, $k a$ would be small compared to unity, and consequently,

and

$$
j_{n}(k a) \cong \text { const. } \times(k a)^{n}
$$

Therefore,

$$
j_{n}^{\prime}(k a) \cong \text { const. } \times(n+1)(k a)^{n-1} .
$$

$$
\begin{aligned}
g_{a} & \simeq \frac{2 \pi(k b)^{2} \operatorname{Re} Z}{\eta_{0}^{2}} \sum_{n=1,3,5}^{\infty} \frac{(2 n+1)}{n(n+1)^{3}}\left(\frac{b}{a}\right)^{2 n}\left[P_{n}^{1}(0)\right]^{2} \\
& \simeq \frac{\pi(k b)^{2} \operatorname{Re} Z}{\eta_{0}^{2}} \frac{3}{4}\left(\frac{b}{a}\right)^{2}\left[1+\frac{7}{32}\left(\frac{b}{a}\right)^{4}+\frac{55}{576}\left(\frac{b}{a}\right)^{8}+\ldots\right] .
\end{aligned}
$$

The efficiency to this approximation is then given by

where

$$
\xi=\frac{1}{1+\left(g_{a} / g_{r}\right)},
$$

$$
g_{a} / g_{r} \cong \frac{\operatorname{Re} Z}{\eta_{0}(k a)^{2}} \frac{9}{2}\left[1+\frac{7}{32}\left(\frac{b}{a}\right)^{4}+\frac{55}{576}\left(\frac{b}{a}\right)^{8}+\ldots\right]
$$

If the material lining the hemispherical cavity had a conductivity $\sigma$, then

$$
\frac{\operatorname{Re} Z}{\eta_{0}} \cong \operatorname{Re}\left[\frac{i \epsilon_{0} \omega}{\sigma+i \epsilon \omega}\right]^{1 / 2} \cong\left(\frac{\epsilon_{0} \omega}{2 \sigma}\right)^{1 / 2} .
$$

For frequencies in the vicinity of $15 \mathrm{kc}$, with a typical ground conductivity of $5 \times 10^{-3} \mathrm{mho} / \mathrm{m}$,

$$
\operatorname{Re} Z / \eta_{0} \cong 0.01
$$

${ }^{6}$ It is also possible to obtain the conductance by integrating the Poynting vector over the area of the slot. 
and, if the cavity radius was $500 \mathrm{ft}, k a \cong 1 / 20$. Therefore,

$$
\begin{aligned}
g_{a} / g_{r} & \cong 18\left[1+\frac{7}{32}\left(\frac{b}{a}\right)^{4}+\ldots\right] \\
& \cong 18 \quad \text { if, say, } \quad b \leq a / 2 .
\end{aligned}
$$

For this case the efficiency, $\xi$, is 0.011 or about 5 percent. This could be improved by increasing the size of the cavity. For example, if the cavity radius was $1,000 \mathrm{ft}$, the efficiency would be about 20 percent for the same wall material.

A more promising approach is to line the cavity wall with a wire mesh. In this case, the surface impedance $Z$ is the parallel combination of the impedance of the wire mesh and the intrinsic impedance of the cavity wall material. It is not difficult to show that [4]

$$
\operatorname{Re} Z / \eta_{0} \cong \frac{G X^{2}}{(G+X)^{2}+G^{2}}
$$

where $G=\sqrt{\frac{\epsilon_{0} \omega}{2 \sigma}} \quad$ and $\quad X=(d / \lambda) \log (d / 2 \pi c)$,

where $d$ is the spacing between the wires, $\lambda$ is the wavelength, and $c$ is the wire radius. For a wire spacing of, say, $13 \mathrm{ft}, d / \lambda \sim 2 \times 10^{-4}$ at $15 \mathrm{kc}$, and for No. 8 wire $2 c \cong 1 / 8 \mathrm{in}$., and therefore $\log (d / 2 \pi c) \sim \log (400) \sim 6.0$. Since $G \cong 0.01$, it follows that

which leads to

$$
\operatorname{Re} Z / \eta_{0} \cong 6.4 \times 10^{-5},
$$

$$
g_{a} / g_{r} \cong 0.115 \text { for } \quad \mathrm{b} \leq a / 2 \quad \text { and } \quad a=500 \mathrm{ft} .
$$

This corresponds to a radiation efficiency of 90 percent for the cavity-backed annular-slot antenna.

No attempt has been made here to estimate the over-all efficiency of the antenna. There is, of course, further absorption of energy radiated into the upper half-space. This loss is due to the finite conductivity of the adjacent ground plane. It could be calculated by representing the annular slot by an equivalent electric dipole and using the available data [4] for loss in radial wire ground systems. The efficiency $\xi$ calculated here is a measure of the performance of the cavity-backed annular slot relative to a more conventional vertical monopole with the same electric moment.

The circular slot concentric to the hemispherical cavity is the simplest model and the most convenient for calculations. It is possible that other geometrical shapes such as elongated or flattened cavities would also be suitable.

The author would like to thank H. L. Knudsen for his helpful suggestions.

\section{References}

[1] H. Levine and J. Schwinger, On the theory of electromagnetic wave diffraction by an aperture in an infinite plane conducting screen, Comm. Pure Appl. Math. 3, No. 4 (1950).

[2] A. Erdelyi, W. Magnus, F. Oberhettinger, and Tricomi, Higher transcendental functions, vol. 2, p. 91 (McGraw-Hill Book Co., Inc., New York, N. Y., 1953). [Note: In eq 21, the right-hand side should read $z^{-1} \sum_{m=0}^{\infty} J_{2 \nu+2 m+1}(2 z)$.J

[3] P. M. Morse and H. Feshbach, Methods of theoretical physics, vol. 2 (McGraw-Hill Book Co., Inc., New York, N. Y., 1953).

[4] J. R. Wait and W. A. Pope, Input resistance of L. F. unipole aerials, Wireless Engineer (May 1955).

Boulder, Colo., August 8, 1957. 